\title{
A VLF Timing Experiment ${ }^{1}$
}

\author{
A. H. Morgan ${ }^{2}$ and O. J. Baltzer ${ }^{3}$
}

(Received April 22, 1964; revised May 22, 1964)

\begin{abstract}
The purpose of the experiment given in this paper was to measure the differential phase stability of two VLF carriers $(19.9 \mathrm{kc} / \mathrm{s}$ and $20.0 \mathrm{kc} / \mathrm{s})$ as received at Austin, Tex., as a function of the observing time, using the former low power standard frequency broadcasts of WWVL, Sunset, Colo.

These measurements indicate that, at the distance involved $(1400 \mathrm{~km})$ and with an averaging time of a few hours, the envelope or group delay variations will cause a "jitter" in the received envelope zeros at the receiver of less than one cycle at $20.0 \mathrm{kc} / \mathrm{s}$. Therefore, a particular cycle of the $20.0 \mathrm{kc} / \mathrm{s}$ carrier as transmitted may be identified at the receiver, thus providing "microsecond" timing.
\end{abstract}

\section{Introduction}

It is well known that the precision of high-frequency $(\mathrm{HF})$ signals is several orders less than that of very low-frequency (VLF) signals due to the severe and unpredictable variations in the propagation medium. This sets an upper limit to the precision with which widely separated clocks may be synchronized using HF timing signals and with which frequency comparisons may be made (fig. 1).

Because of their high phase stability and low attenuation rates, VLF signals are well suited for the dissemination of standard frequencies. This was the basis for changing to their use in 1961 in calibrating and controlling the frequencies as broadcast at WWV. The marked improvement that resulted is clearly evident in figure 1 , where $S^{2}$ is the sample variance and $S$ the sample standard deviation of the mean, in parts in $10^{10}$. Further evidence in the high stability of LF and VLF signals is given in figure 2, which shows the excellent agreement of the signals of the NBS standard LF (WWVB) and VLF (WWVL) stations as received at WWV.

If the precisions of frequency comparison of a few parts in $10^{11}$ [Pierce, 1958] were obtainable in the dissemination of time signals, it would permit synchronization of clocks to $1 \mu$ sec over wide areas of the world. Because of the narrow bandwidths available at VLF and the high $Q$ factors of the antennas, it has not been possible to use sharply rising pulses as precise time markers.

This appears to be a basic limitation, and if so, it could not be circumvented by trick circuitry. In any case, the bandwidths needed for such pulses are simply not available. Moreover, even if they were, it appears that the high ambient noise levels at VLF would be a severe problem in the effective use of wideband systems.

\footnotetext{
1 This paper was given at the VLF Symposium in Boulder, Colo., August 13 , 1963.

2 Radio Standards Laboratory, National Bureau of Standards, Boulder Laboratories, Boulder, Colo.

3 Senior Vice President, Tracor, Inc., Austin, Tex.
}

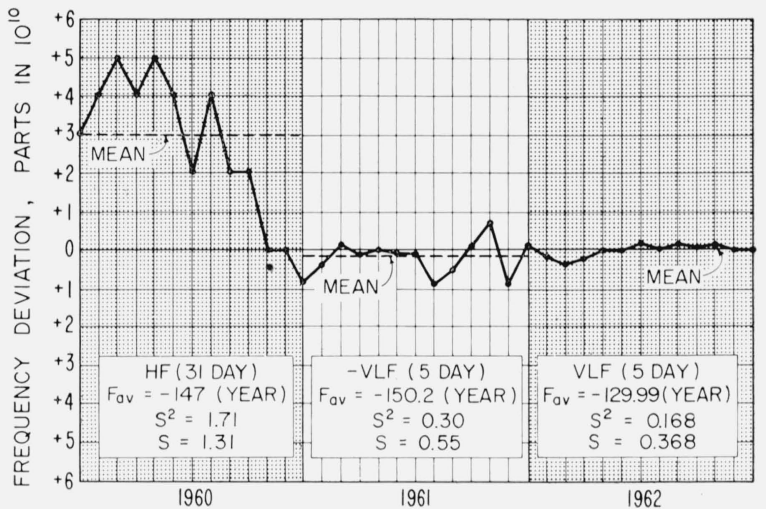

Figure 1. Improvements in the frequency control of $W W V$. The units of $S$ are in parts in $10^{10}$.

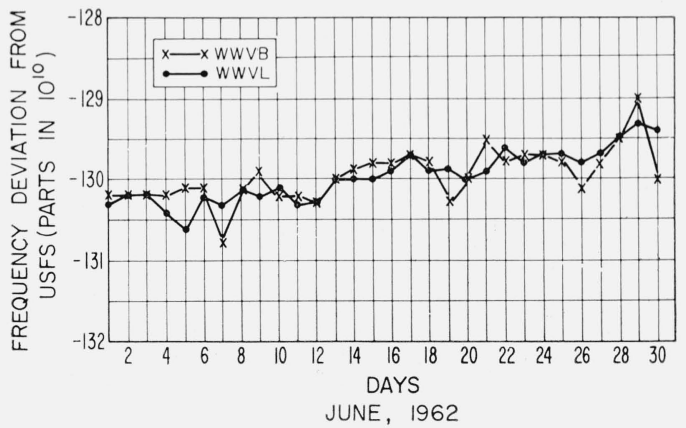

Figure 2. Agreement of values of frequency of $W W V L$ and $W W V B$ as received at $W W V$.

The positive zero crossovers of the carrier wave, however, do provide the desired precision for timing marks, but at $20 \mathrm{kc} / \mathrm{s}$, for instance, there are ambiguities in the time every $50 \mu$ sec. To resolve these ambiguities another carrier frequency, $19.9 \mathrm{kc} / \mathrm{s}$, for example, may be transmitted every second. The much more widely spaced zero crossovers of the received envelope serve as coarse time markers to identify certain cycles of the carrier. They, in turn, 
may be identified by reference to other time scales such as the signals of WWV.

However, high differential phase stability is necessary in order to be able to identify the correct carrier cycle with this method.

The group delay of these signals, as given in a previous paper [Watt et al., 1961] is

$$
t_{d}=\frac{\phi_{2}-\phi_{1}}{\omega_{2}-\omega_{1}}
$$

where $\phi=\omega t$. As pointed out in the previous paper the received phase, $\phi$, at each carrier frequency may consist of a constant term, $\phi^{\prime}$, and a fluctuating term, $\pm \delta \phi^{\prime}$, due to effects of the propagation medium; i.e., $\phi=\phi^{\prime} \pm \delta \phi^{\prime}$. This would also indicate that the group delay time could be similarily expressed; i.e., $t_{d}=t_{d}^{\prime}$ $\pm \delta t_{d}^{\prime}$. If these expressions are put into (1), the result is

$$
t_{d}^{\prime} \pm \delta t_{d}^{\prime}=\frac{\left(\phi_{2}^{\prime}-\phi_{1}^{\prime}\right) \pm\left(\delta \phi_{2}^{\prime}-\delta \phi_{1}^{\prime}\right)}{\omega_{2}-\omega_{1}}
$$

The differential phase is the quantity of interest, and is given as

$$
\delta t_{d}^{\prime}=\frac{\left(\delta \phi_{2}^{\prime}-\delta \phi_{1}^{\prime}\right)}{\left(\omega_{2}-\omega_{1}\right)}
$$

or, briefly, as

$$
\Delta\left(\delta \phi^{\prime}\right)=\Delta \omega \delta t_{d}^{\prime}
$$

where

$$
\Delta\left(\delta \phi^{\prime}\right) \equiv\left(\delta \phi_{2}^{\prime}-\delta \phi_{1}^{\prime}\right) \text { and } \Delta \omega \equiv\left(\omega_{2}-\omega_{1}\right)
$$

In the previous paper a discussion was given of the basic limitations in the stability of the VLF signals, including path phase distortion, carrier-to-noise, and group delay variations as related to the time dissemination problem.

\section{Purpose of the Experiment}

The purpose of this experiment was to measure the differential phase stability of two VLF carriers $(19.9 \mathrm{kc} / \mathrm{s}$ and $20.0 \mathrm{kc} / \mathrm{s})$ as received at Austin, Tex., as a function of the length of the observing time. These results were obtained by using the former low power standard frequency broadcasts of WWVL, Sunset, Colo.

It may be shown from (1) that a change in the phase of zeros of either carrier wave as received will cause a change in the antiphase points that is larger by a factor equal to the ratio of the carrier frequency (which everyone is chosen for the timing) to the envelope frequency. In this case (at $20 \mathrm{kc} / \mathrm{s}$ ) the factor is $20,000 \mathrm{c} / \mathrm{s} / 100 \mathrm{c} / \mathrm{s}=200$.

\section{Description of the Experiment}

Such signals, phase locked [Fey et al. 1962] to the working frequency standard (fig. 3) by means of a VHF radio link to eliminate the effects of phase shifts in the VLF antenna system and transmitter, were transmitted from Sunset, Colo., and received at Austin, Tex., a distance of about $1400 \mathrm{~km}$. The receiving equipment used consisted of two phaselocked receivers, one at each carrier frequency, with provision to record a voltage analog of the received phase of each.

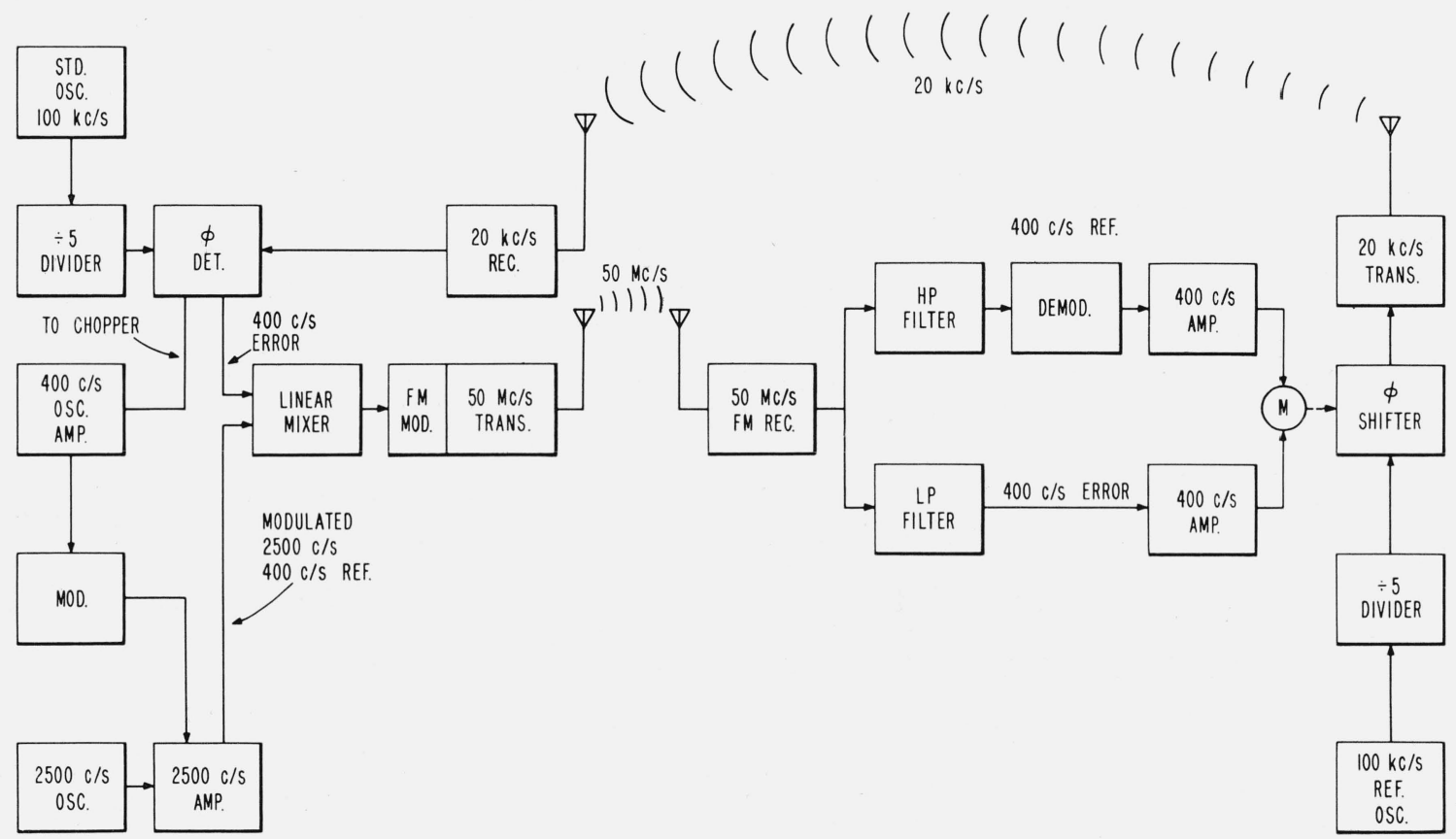

Figure 3. Sunset phase-lock system for WWVL (20 kc/s). 
A simplified block diagram of a typical phase-lock receiver is shown in figure 4. As may be seen, the signal available to the user is not the received signal but one locally generated and kept in phase lock with it. Therefore, the receiver output signal is: (1) very constant in amplitude and (2) relatively free of noise perturbations, both very important considerations. The receiving system acts as a very narrow band filter (bandwidths of 0.01 to 0.001 cycle) which permits use of signals several tens of decibels below the noise in a $1 \mathrm{kc} / \mathrm{s}$ bandwidth.

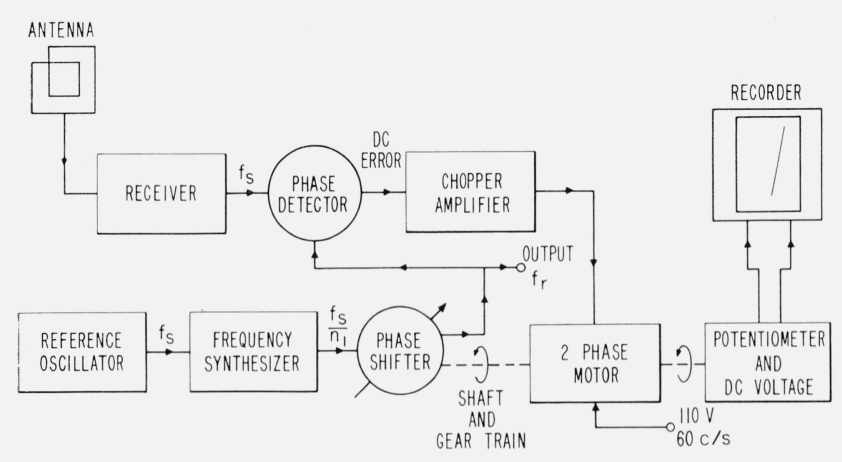

Figure 4. Phase-lock receiving system.
A block diagram of the receiving system used is shown in figure 5. It may be seen that a differential phase meter was also included to record continuously the differences in phase of the two signals. A typical set of records is shown in figure 6 , where the lower parallel traces represent the received phase of the 20.0 and $19.9 \mathrm{kc} / \mathrm{s}$ carriers. That the traces of the two signals follows each other very closely is quite apparent. The upper trace is the magnified phase difference between the two carriers.

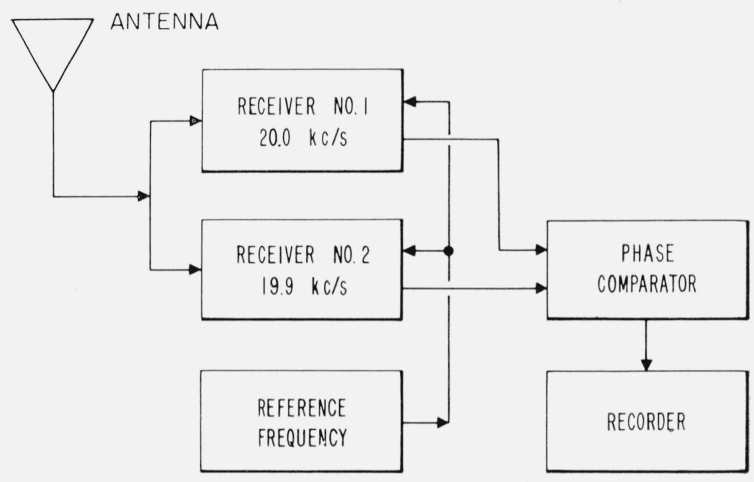

Figure 5. Diagram of receiving system at Austin, Tex.
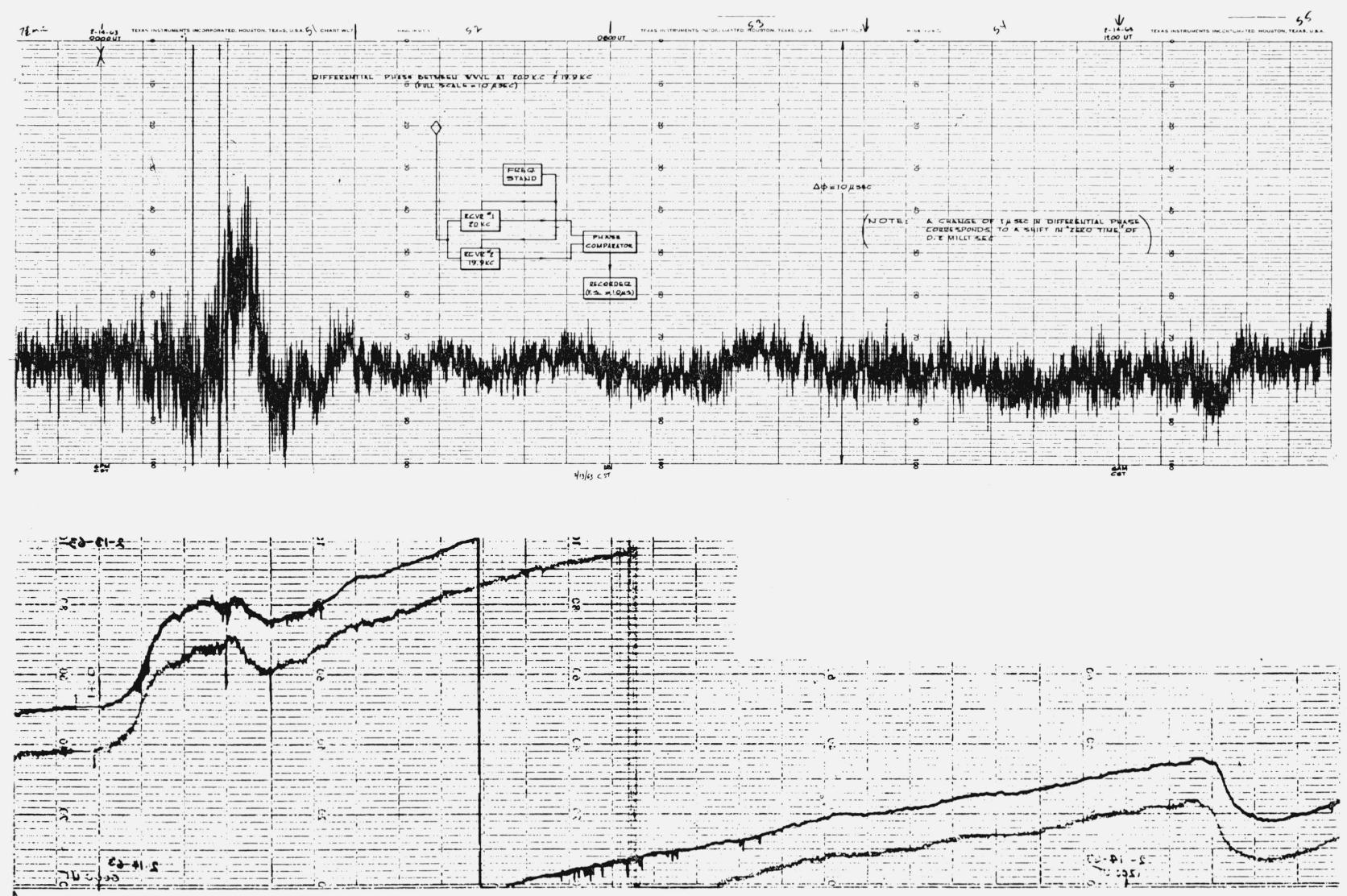

Figure 6. Typical set of recordings, February 1963. 


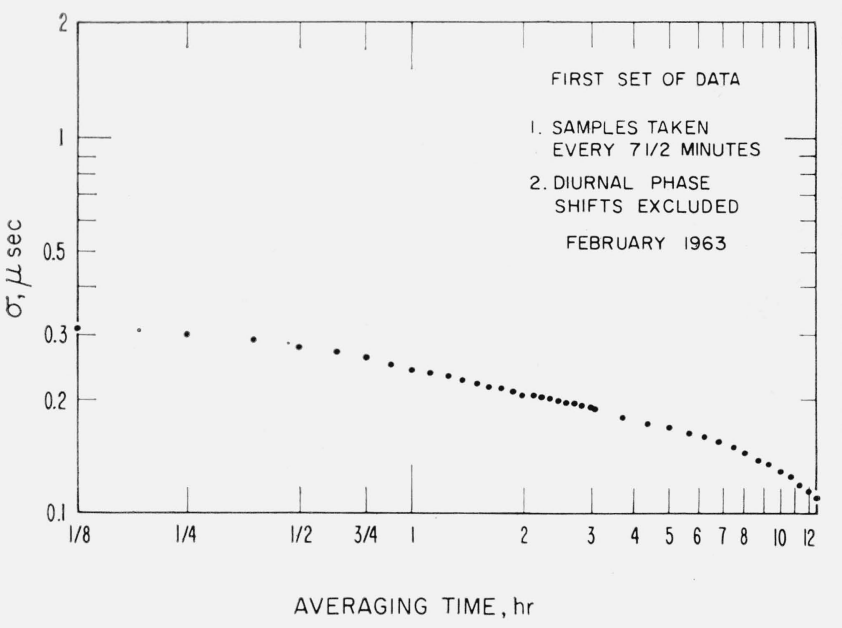

Figure 7. Differential phase stability.

(Sampling time $=7.5 \mathrm{~min}$.)

\section{Preliminary Results Obtained}

Two sets of several days recordings, as described above, were made at Austin, Tex., and the data analyzed.

For analysis by a digital computer, the data on the differential phase records were scaled at intervals of $7 \frac{1}{2} \mathrm{~min}$ for the first set of records and at 5-min intervals for the second set, but excluding periods of diurnal phase shifts. The calculated results are shown in figures 7 and 8 . They are a plot of the sample standard deviation, in microseconds, as a function of the averaging time in seconds, of the differential phase converted to differential time, as given in (3), between the 19.9 and $20.0 \mathrm{kc} / \mathrm{s}$ signals as received in Austin, Tex.

The results given in figures 7 and 8 indicate that, with an averaging time of a few hours, the envelope or group delay variations will cause a "jitter" in the envelope zeros at the receiver of about $\pm 50 \mu \mathrm{sec}$, or about 1 cycle at $20 \mathrm{kc} / \mathrm{s}$, during periods of no diurnal phase change. This means that a particular cycle

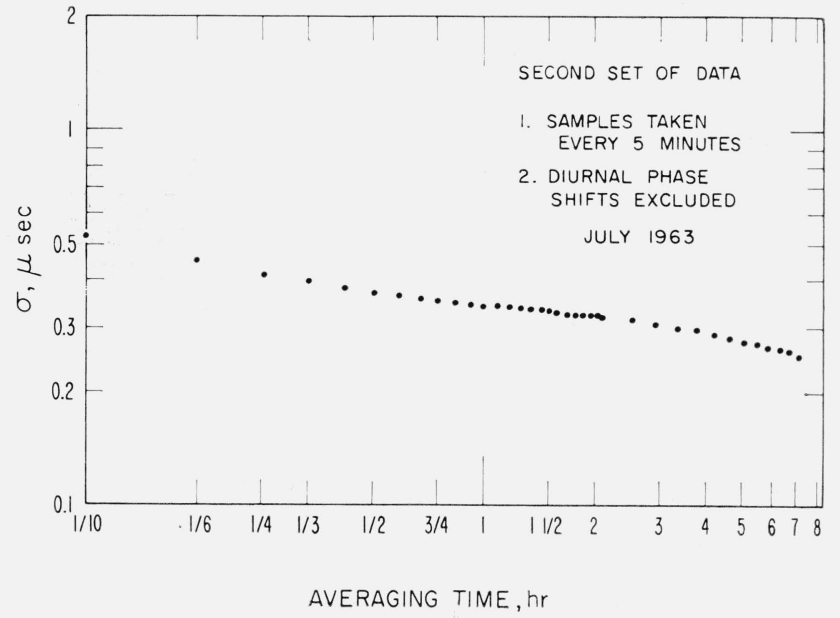

Figure 8. Differential phase stability. (Sampling time $=5.0 \mathrm{~min}$.)

of the carrier as transmitted may be identified at Austin, Tex., provided the group delay time over the path is known. In that case, a precise clock in Austin could be synchronized to one in Boulder to "microsecond" accuracy using the zero crossovers of the $20.0 \mathrm{kc} / \mathrm{s}$ signals.

The contributions of L. Fey, E. Marovich, and E. L. Crow are hereby acknowledged. Also, the manuscript was typed by Miss C. A. Merkling.

\section{References}

Fey, R. L., J. B. Milton, and A. H. Morgan (March 17, 1962), Remote phase control of radio station WWVL, Nature, 193. No. 4820, 1063-1064.

Pierce, J. A. (December 1958), Recent long-distance frequency comparisons, IRE Trans. Instr.I-\%, No.3-4, 207-210. Watt, A. D., R. W. Plush, W. W. Brown, and A. H. Morgan (1961), J. Res. NBS 65D (Radio Prop.) No. 6, 617-627.

(Paper 68D11-423) 